\title{
Integrable maps with non-trivial topology: application to divertor configurations
}

\author{
T. Kroetz ${ }^{1}$, M. Roberto ${ }^{1}$, I.L. Caldas ${ }^{2}$, R.L. Viana ${ }^{3,6}$, \\ P.J. Morrison ${ }^{4}$ and P. Abbamonte ${ }^{5}$ \\ ${ }^{1}$ Instituto Tecnológico de Aeronáutica, Centro Técnico Aeroespacial, Departamento de \\ Física, 12228-900, São José dos Campos, São Paulo, Brazil \\ ${ }^{2}$ Instituto de Física, Universidade de São Paulo, 05315-970, São Paulo, São Paulo, Brazil \\ ${ }^{3}$ Departamento de Física, Universidade Federal do Paraná, 81531-990, Curitiba, Paraná, \\ Brazil \\ ${ }^{4}$ Department of Physics and Institute for Fusion Studies, The University of Texas at Austin, \\ Austin, TX 78712, USA \\ ${ }^{5}$ Department of Physics, University of Illinois, Urbana, IL 61801-3080, USA \\ E-mail: viana@fisica.ufpr.br
}

Received 14 May 2009, accepted for publication 9 September 2009

Published 23 February 2010

Online at stacks.iop.org/NF/50/034003

\begin{abstract}
We explore a method for constructing two-dimensional area-preserving, integrable maps associated with Hamiltonian systems, with a given set of fixed points and given invariant curves. The method is used to find an integrable Poincaré map for the field lines in a large aspect ratio tokamak with a poloidal single-null divertor. The divertor field is a superposition of a magnetohydrodynamic equilibrium with an arbitrarily chosen safety factor profile, with a wire carrying an electric current to create an $X$-point. This integrable map is perturbed by an impulsive perturbation that describes non-axisymmetric magnetic resonances at the plasma edge. The non-integrable perturbed map is applied to study the structure of the open field lines in the scrape-off layer, reproducing the main transport features obtained by integrating numerically the magnetic field line equations, such as the connection lengths and magnetic footprints on the divertor plate.
\end{abstract}

PACS numbers: $52.55 . \mathrm{Rk}, 52.55 . \mathrm{Dy}, 05.45 . \mathrm{Pq}$

(Some figures in this article are in colour only in the electronic version)

\section{Introduction}

The divertor is an essential component of modern tokamak design [1]. In essence, a divertor is a shaped metallic plate that is suitably placed outside the plasma boundary so as to capture or divert particles escaping from the plasma. There are two basic roles a divertor plays in tokamak design: (i) it exhausts particles coming both from fusion products, such as He atoms or impurity atoms stemming from plasma-wall interactions and (ii) it removes heat from the plasma discharge. For example, the divertor of ITER is designed to withstand a heat load of 5-10 $\mathrm{MW} \mathrm{m}^{-2}$ coming from alpha particles resulting from fusion reactions taking place in the plasma core $[2,3]$.

Basic mechanisms underlying the operation of a divertor are also examples of the practical application of chaos theory in plasma physics [4]. In tokamaks, plasma is confined in magnetic field configurations with nested flux surfaces that

\footnotetext{
6 Author to whom any correspondence should be addressed.
}

play the role of invariant curves of a Hamiltonian system [5-7]. These nested curves are surrounded by a region of chaotic field lines, which occupy the outer plasma region extending from the plasma boundary to the tokamak inner wall, including the so-called scrape-off layer [5]. Chaotic behaviour exists in the following sense: two (infinitesimally) nearby field lines depart from each other at a positive exponential rate after toroidal revolutions (where the toroidal angle plays the role of time in the Hamiltonian theory) [6, 7].

In a first approximation, charged particles follow magnetic field lines, and since the divertor plate is placed to intersect a significant portion of the chaotic region, many particles escaping from the plasma will eventually hit the divertor [8-10]. In spite of its simplicity, the divertor concept still raises a series of important questions in both technological and theoretical aspects. For example, the particle and heat deposition in the divertor plates is not uniform, rather presenting regions of high and low deposition, sometimes revealing potentially dangerous places of intense localized 
loading. Moreover, these high deposition layers possess a fractal-like structure, called magnetic footprints [11-13], which have also been experimentally investigated [14, 15].

Only recently have such magnetic footprints been explained by the underlying mathematical structure of the outer chaotic region [14-18]. The latter is not uniform, as might be expected, and it possesses escape channels due to the complicated structure of invariant manifolds attached to the unstable orbits embedded in the chaotic region. An investigation of the divertor chaotic region demands fast and efficient numerical procedures for the tracking of a typically huge number of orbit points. Direct attacks using numerical integration of the differential equations of the magnetic field lines are computationally time consuming. Moreover, to preserve magnetic flux conservation such computer codes must be symplectic, which makes them even more difficult to apply in investigations of a more fundamental nature [19-22].

Discrete maps have been extensively employed to investigate the field line properties in a tokamak with a divertor. The unperturbed magnetic field of a tokamak with a divertor was modelled in [19] by considering two infinitely long parallel wires, and the resulting dependence of the width of the scrapeoff layer on the error fields was investigated. The magnetic field line equation for the two-wire divertor field perturbed by a non-axisymmetric field was numerically integrated in [20,21] in order to study the structure of the open field lines and their connection lengths between two divertor plates. A field line equation integration code was used in $[17,22]$ to study the magnetic flux loss through the edge of a poloidally diverted tokamak and also to show how the structure of the homoclinic tangle near the separatrix ultimately determines the location and structure of magnetic footprints and heat buildup on the tokamak wall.

On the other hand, discrete time Poincaré maps are much faster to iterate and can yield reliable results on key features of divertor phenomenology, such as magnetic footprints and escape basins present in outer chaotic magnetic configurations in tokamaks $[4,23,25]$. In fact, in the past two decades many works on divertor physics have used discrete maps of various types and geometries [11,12,26-29]. A series of symplectic maps for divertor fields were proposed in [30-37] to investigate the effect of different kinds of resonant perturbations on the field line topology. These maps provide a generic qualitative description of field line dynamics in a single-null divertor configuration and are useful for studying the field line deposition on the collector plates. However, the parameters of such maps do not correspond to the control parameters that can be directly reached in real divertors. Although relevant generic constraints are considered in proposing these maps, their parameters do not correspond to the control parameters that can be directly reached in real divertors, since they were not obtained from the magnetic field equations.

A field line Hamiltonian was used as the generating function for a map that describes a superposition of a piecewise analytic representation of the measured poloidal flux function with the magnetic perturbation measured in the DIII-D tokamak to model divertor experimental data in DIII-D [27]. This approach was further generalized by including an MHD analytic equilibrium so as to obtain the generating function [28]. On the other hand, canonical maps were derived from magnetic field line equations, to investigate field line trajectories near the $X$-point in poloidal divertor tokamaks $[11,38]$. These maps are constructed from generalized Poincaré integrals by a method described in [39], and have been generalized for a more complex geometry consisting of a three wire plasma model considering the toroidal effect of the equilibrium [29,40]. Moreover, external resonant magnetic perturbations to the divertor map were also included in this framework [41]. Recently, a field line convection coefficient was added to this approach to estimate the chaotic transport near the magnetic separatrix $[12,41]$. Another canonical procedure to derive a map from magnetic field line equations was introduced in [26], where it was studied using parameters of the tokamak COMPASS-D.

In this paper, we construct a symplectic divertor map using a general method developed to tailor symplectic maps with specified stationary points [42]. For a single-null divertor, this stationary point is a saddle (or $X$-point) embedded in a chaotic region and placed between the plasma boundary and the divertor plate. Firstly, we use the method to obtain an integrable map compatible with the single null, and then we perturb the system in a non-integrable fashion by adding the effect of an ergodic limiter. Starting from an integrable map allows us to accurately control the onset and development of chaos. With suitable perturbation, a large chaotic region that intersects the divertor plate is created, which enables us to investigate the formation of magnetic footprints and their fractal properties.

The rest of this paper is organized as follows: in section 2 we outline the general method used to obtain integrable maps. In sections 3 and 4, we apply the method to a magnetic field line configuration with a single null. The addition of a nonintegrable perturbation caused by an ergodic limiter and the resulting divertor map are treated in section 5. In section 6 we present results on the fractal structure of magnetic footprints, using the divertor map we have derived. Our conclusions are left to the last section.

\section{Hamiltonian systems and symplectic maps}

Surfaces of section (also called Poincáre maps) are useful for viewing cross-sections of general phase space trajectories. In the present context, they show collections of points in the plane that can reveal important topological features of the trajectories and energy surfaces when the system is conservative. When the equations describing the time evolution of the trajectories are ordinary differential equations with bounded energy surfaces, the coordinates of each point in a given surface of section, written as $\left(x_{n}, y_{n}\right)$, are uniquely determined by the coordinates of the preceding point, yielding a map

$$
\begin{aligned}
& x_{n+1}=f\left(x_{n}, y_{n}\right), \\
& y_{n+1}=g\left(x_{n}, y_{n}\right),
\end{aligned}
$$

where the functions $f$ and $g$ are only explicitly known under the fortuitous circumstance that the trajectories derived from a Hamiltonian function can be analytically solved [43].

After initial conditions are chosen in a specified region of the surface of section, the map equations (1) and (2) can be iterated to generate trajectories for an arbitrarily length of time. 
The accuracy of the generated orbit is thus only influenced by one-step numerical error, and this allows us to track a large number of trajectories in phase space with controlled numerical accuracy. Hence, Poincaré maps are more efficient for viewing phase space topology (especially when looking at many different trajectories over long times) than finding the surface of section by numerically integrating the dynamical equations.

Because Hamiltonian systems preserve integral invariants, an example being phase space volume preservation (Liouville's theorem), a map of the form (1) and (2) must be symplectic, i.e. it will have a unit Jacobian determinant,

$$
[f, g]=\frac{\partial f}{\partial x_{n}} \frac{\partial g}{\partial y_{n}}-\frac{\partial f}{\partial y_{n}} \frac{\partial g}{\partial x_{n}}=1 .
$$

This is a rather stringent property that cannot be taken for granted, in general, when the map is obtained directly from Hamiltonian differential equations of motion.

As a simple, but representative example, consider the general one degree-of-freedom Hamiltonian system with canonical coordinates $(x, y)$, with $y$ being the canonical momentum conjugate to the coordinate $x$. Given the Hamiltonian $H(x, y)$, the equations of motion are

$$
\begin{gathered}
\frac{\mathrm{d} x}{\mathrm{~d} t}=\frac{\partial H}{\partial y}, \\
\frac{\mathrm{d} y}{\mathrm{~d} t}=-\frac{\partial H}{\partial x},
\end{gathered}
$$

whence it is seen that $H$ is a constant of motion. Because the number of constants of motion matches the number of degrees of freedom, all one degree-of-freedom Hamiltonian systems are integrable in the sense of Liouville. This means trajectories lie on contours of $H$ and that there exists a canonical change of coordinates $(x, y) \leftrightarrow(\theta, J)$ such that in the new coordinates the Hamiltonian depends only on $J$, i.e. $H(x, y)=\bar{H}(J)$. Here the action variable $J$ is defined by $J=\oint y \mathrm{~d} x / 2 \pi$, where $y$ is written as a function of $x$ by setting $H$ equal to a constant and $\theta$ is obtain from the requirement $[\theta, J]=1$. Thus, (4) and (5) in the new coordinates become

$$
\begin{gathered}
\frac{\mathrm{d} \theta}{\mathrm{d} t}=\frac{\partial \bar{H}}{\partial J}, \\
\frac{\mathrm{d} J}{\mathrm{~d} t}=0,
\end{gathered}
$$

which have the simple solution $\theta=\theta_{0}+\Omega(J) t$ and $J=J_{0}$ with initial condition $\left(\theta_{0}, J_{0}\right)$ and $\Omega(J):=\partial \bar{H} / \partial J$. In the coordinates $(\theta, J)$ one can easily obtain explicitly the integrable map that takes any initial point to a point at any future time $\Delta t$ as follows:

$$
\theta_{n+1}=\theta_{n}+\Omega\left(J_{n+1}\right) \Delta t \quad \text { and } \quad J_{n+1}=J_{n} .
$$

Thus, the situation appears to be quite simple; although one can rarely obtain the transformation $(x, y) \leftrightarrow(\theta, J)$ in closed form, a quadrature integral is at hand, but numerical implementation requires iteration. Since the transformation from 'time' $n$ to $n+1$ is generated by Hamilton's equations, this mapping must be area preserving. We have chosen $\Omega$ to be a function of $J_{n+1}$ rather than $J_{n}$, so that the area preserving property (3) takes a particularly simple form.

When the plane is foliated by closed nested contours the action transformation is uniquely defined, but when the contours of $H$ have more interesting topology with multiple contours corresponding to the same value of $H$, then the transformation $(x, y) \leftrightarrow(\theta, J)$ becomes multibranched, and invertibility of the transformation requires the addition of a discrete branching index, say $\mathcal{I}$. As we see later, the field line 'dynamics' of a tokamak equilibrium with a singlenull divertor constitutes an example of a Hamiltonian system with this branching. For a general one degree-of-freedom Hamiltonian system, one has an integrable area-preserving map of the two-dimensional phase space by effecting the following procedure: choose an initial point $\left(x_{n}, y_{n}\right)$; this point determines the index $\mathcal{I}$ and an initial value of the energy, whence one can obtain coordinates $\left(\theta_{n}, J_{n}\right)$; from (8) one then obtains $\left(\theta_{n+1}, J_{n+1}\right)$; finally, given $\left(\theta_{n+1}, J_{n+1}\right)$ and $\mathcal{I}$, one obtains $\left(x_{n+1}, y_{n+1}\right)$.

Because the procedure above is cumbersome in general, we base our construction of integrable maps on the Hamiltonian for one-dimensional motion of a particle of unit mass under a potential $V(x)$, namely

$$
H(x, y)=\frac{y^{2}}{2}+V(x),
$$

with the equations of motion

$$
\begin{gathered}
\frac{\mathrm{d} x}{\mathrm{~d} t}=y, \\
\frac{\mathrm{d} y}{\mathrm{~d} t}=-V^{\prime}(x) .
\end{gathered}
$$

This form of $H$ does not allow for general energy contours, but it is sufficient for obtaining symplectic maps with the features desired here, i.e. maps with a given unstable fixed point with an invariant curve of a homoclinic type. The 'trajectory integration method', described in essence above, together with the example using the Hamiltonian (9) was introduced in [42]. The virtue of using the Hamiltonian (9) lies in the fact that the discretization procedure can be effected in simpler form, alleviating the need for interaction, and thus produces no modelling error in the sense that the map still describes an integrable system with all trajectories confined to invariant curves. If one wishes to obtain a map with a set of fixed points lying on a line (without loss of generality, the $x$-axis) and with invariant curves having a desired topology, then one can proceed as follows:

1. write down a one-dimensional potential $V(x)$ that produces a phase space portrait with the same fixed points and topology as the desired mapping equations;

2. integrate the canonical equations (10) and (11) so as to express analytically the coordinates $x$ and $y$ in terms of their initial conditions $\left(x_{0}, y_{0}\right)$ and the time $t$;

3. turn the continuous time parameter $t$ into a discrete time step $\Delta t$.

As a result, one obtains an integrable map with the same fixed points as those determined by the potential $V(x)$. This is, of course, equivalent to a special case of the coordinate change procedure described above. 
A particularly tractable example is the harmonic oscillator which has the potential $V(x)=x^{2} / 2$ and Hamiltonian $H(x, y)=y^{2} / 2+x^{2} / 2$. The corresponding dynamical equations can be easily integrated and yields the linear (and trivially integrable) map

$$
\begin{gathered}
x_{n+1}=x_{n} \cos \Delta+y_{n} \sin \Delta, \\
y_{n+1}=-x_{n} \sin \Delta+y_{n} \cos \Delta,
\end{gathered}
$$

where the time step $\Delta$ may be chosen arbitrarily. In section 3 we use this simple example to construct something more interesting. Other potentials could have equally been chosen. For example, another combination of local one-dimensional harmonic oscillators (for which the potential function is a combination of parabolae spliced together so that their slopes match at the connection points) would yield vertically elongated and horizontally displaced invariant curves. If such potentials were used to generate invariant curves, then the corresponding Hamiltonian equations would be more complicated.

There exists another situation where explicit maps can be obtained exactly, namely when there is a periodic forcing that is localized in time. This occurs, for example, when the potential $V$ is time dependent and possesses a series of periodic 'kicks' and is given by

$$
V(x, t)=\hat{V}(x) \sum_{n=-\infty}^{\infty} \delta(t-n \tau),
$$

where $\delta(t)$ is the Dirac delta function. Integration of (14) across a kick yields the map. For potentials that are periodic in time and nearly non-zero for only narrow intervals, this is a reasonable approximation. However, sometimes this discretization procedure is used when the potential is not close to the kick form, but in fact is even constant in time. When this is done it amounts to a finite difference scheme, and for larger $\Delta t$ it introduces error into the system that usually appears in the form of chaos in the trajectories. As a consequence when used in this manner, even for small $\Delta t$, the fixed points of the obtained discrete map are only approximations of those implied by the shape of the potential $V(x)$. In section 5 we use a kick map of physical origin for introducing chaos into our divertor model.

\section{Integrable map for a single-null divertor}

As mentioned above, the magnetic field line equations for confined plasmas with axisymmetry can be described as Hamiltonian systems (see, e.g. [5-7, 23-25]). In our case, for unperturbed flux surfaces with the topology of closed nested tori, the Poincare surface of section is an actual poloidal plane intersecting the torus at a fixed constant toroidal angle $\phi$. Due to the axisymmetry of equilibrium states in such devices, this angle is a cyclic coordinate and plays the role of time in the Hamiltonian description of a field line, with the other two variables being the canonical coordinates. Thus, the field line equations can be cast in the form of Hamilton's equations describing a single degree-of-freedom integrable system.

We choose a simple coordinate system that retains the essential features we expect in more realistic geometries. We

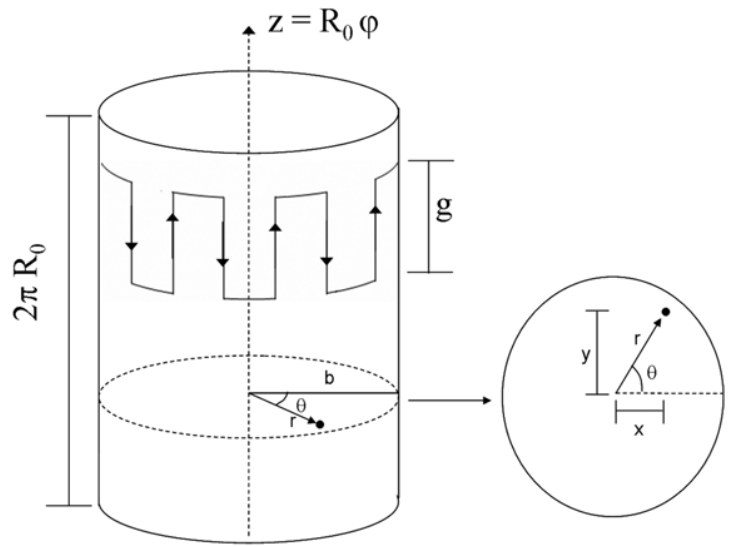

Figure 1. Schematic view of a periodic cylinder approximation of a tokamak toroidal chamber. The polar and rectangular coordinates are also shown in the figure in a circular section of the tokamak, as well as ergodic limiter configuration with the arrows indicating the current direction. The ring-shaped coil has length $g$.

start from a toroidal flux surface of minor and major radii given by $b$ and $R_{0}$, respectively. If the aspect ratio $R_{0} / b$ is big, then the periodic cylinder of length $2 \pi R_{0}$, where the magnetic axis is parametrized by the coordinate $z=R_{0} \phi$, is a good approximation. Moreover, in this case the equilibrium toroidal field $B_{z}=B_{0}$ is nearly uniform and hence torodicity can be neglected in the lowest-order approximation. Accordingly, the position of a point in the surface of section can be described by cylindrical coordinates $(r, \theta, z)$, as sketched in figure 1 .

Alternatively, a rectangular system can be used with the coordinates: $x=r \cos \theta$ and $y=r \sin \theta$, with the tokamak wall characterized by $x^{2}+y^{2}=b^{2}$. The equation for the equilibrium magnetic field in such large aspect ratio tokamaks is

$$
\boldsymbol{B}(x, y)=B_{0} \hat{z}+\nabla \psi(x, y) \times \hat{z} .
$$

Therefore, regarding the spatial coordinates $x$ and $y$ as canonically conjugate variables, with the $z$-coordinate playing the role of time, the magnetic field line equations can be written as Hamilton equations

$$
\begin{gathered}
\frac{\mathrm{d} x}{\mathrm{~d} z}=\frac{\partial \psi}{\partial y}, \\
\frac{\mathrm{d} y}{\mathrm{~d} z}=-\frac{\partial \psi}{\partial x},
\end{gathered}
$$

with the flux function $\psi(x, y)$ as the Hamiltonian. Since $\psi$ is a constant of motion for axisymmetric systems, the system is integrable and a map can be constructed by the procedures described in section 2 .

We first present a simple integrable map for the bulk plasma. Accordingly, we associate the flux function with the harmonic oscillator Hamiltonian

$$
\psi(x, y)=\frac{1}{2} y^{2}+\frac{1}{2} x^{2},
$$

resulting in the map of equations (12) and (13). At first glance, this choice would seem too specific and even too trivial to explain an actual tokamak equilibrium field. However, since the argument of the sines and cosines in the map of (12) and (13) 


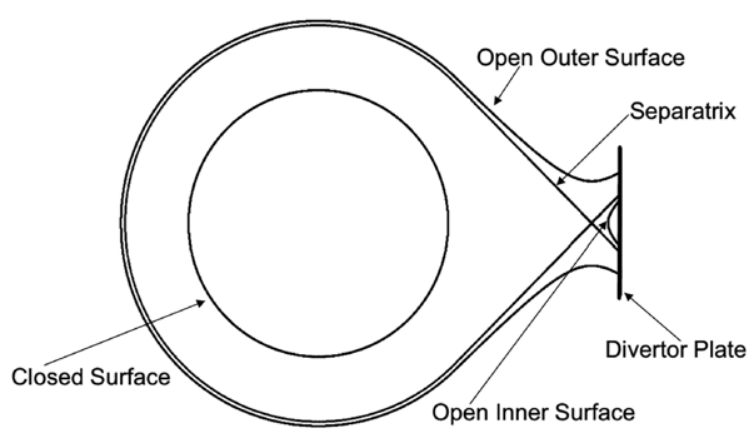

(a)

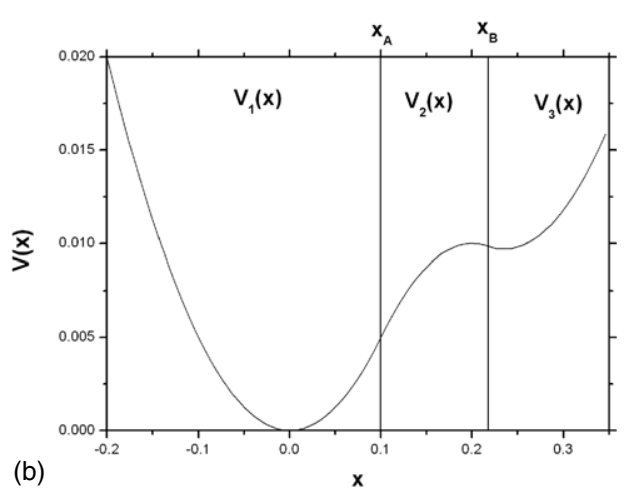

Figure 2. (a) Scheme of a single-null divertor. (b) One-dimensional potential for the divertor map, for $c=0.200$ and $a=0.235$.

has no radial dependence, as one travels from the origin radially outwards, the safety factor of the corresponding flux surfaces, $q(r)=2 \pi / \Delta$, is a constant. Since each surface is the locus of points with the same value of the Hamiltonian function $\psi(r)=r^{2} / 2$, the flux surface radius is $r=\sqrt{2 \psi}$. Therefore, to give $q$ the desired $r$-dependence, one needs only to give the discretization parameter a $\psi$-dependence. Map (12) and (13) can be changed by allowing $\Delta$ to be a function of $\psi$. In this way we can describe the equilibrium structure of a plasma with an arbitrary safety factor radial profile $q(r)$. Once we know the latter it is possible to choose the time step $\Delta(r)=2 \pi / q(r)$ for each value of $r$. However, this simple relationship between $\Delta$ and $q$ is only valid for circular surfaces. For other surface topologies, $\Delta$ may depend on $q$ in more complicated ways.

The trajectory integration method can also be used to derive an explicit, integrable map for the field lines in a tokamak with a single-null divertor. As stated before, the prerequisite for use of the trajectory method is that all the fixed points lie on the same line (e.g. the $x$-axis). In a singlenull divertor, the magnetic axis and the hyperbolic saddle $(X$-point) are the fixed points of interest and the straight line connecting them is also the symmetry line for the divertor plate (figure 2(a)). A one-dimensional potential function that produces this topology has two wells with a barrier with a maximum located between the divertor plate and the plasma boundary. To simply achieve this configuration we can use three parabolas spliced together so that their slopes match at the connection points [42],

$$
V(x)= \begin{cases}\frac{1}{2} x^{2}, & x<x_{A}, \\ -\frac{1}{2}(x-c)^{2}+d, & x_{A} \leqslant x \leqslant x_{B}, \\ \frac{1}{2}(x-a)^{2}+b, & x>x_{B},\end{cases}
$$

where there are two chosen parameters $a$ and $c$. The parameters $x_{A}=c / 2$ and $x_{B}=(c+a) / 2$ are the connection points, with $b=\left(2 a c-a^{2}\right) / 4$ and $d=c^{2} / 4$ being parameters adjusted to make the parabolas join smoothly (figure $2(b)$ ). The two elliptic points are at $x=0$ and $x=a$ and the hyperbolic point is at $x=c$. All these points have $y=0$.

We can obtain the field line trajectories for each parabolic function by the general procedure outlined in the previous section. Accordingly, we turn the time parameter $t$ into a discrete time step $\Delta$. This yields a map for each region of the potential (19):

$x_{n+1}=\left\{\begin{array}{lc}x_{n} \cos \Delta+y_{n} \sin \Delta, & x_{n}<x_{A}, \\ \left(x_{n}-c\right) \cosh \Delta+y_{n} \sinh \Delta+c, & x_{A} \leqslant x_{n} \leqslant x_{B}, \\ \left(x_{n}-a\right) \cos \Delta+y_{n} \sin \Delta+a, & x_{n}>x_{B},\end{array}\right.$

$y_{n+1}= \begin{cases}-x_{n} \sin \Delta+y_{n} \cos \Delta, & x_{n}<x_{A}, \\ \left(x_{n}-c\right) \sinh \Delta+y_{n} \cosh \Delta, & x_{A} \leqslant x_{n} \leqslant x_{B}, \\ -\left(x_{n}-a\right) \sin \Delta+y_{n} \cos \Delta & x_{n}>x_{B} .\end{cases}$

The field line trajectories lie on circles for the first and third regions, and hyperbolae in the second region. We are interested, in particular, in the lines that stem from the $X$-point (its invariant manifolds). The circles are expected to join the hyperbolae so as to describe flux surfaces that confine the tokamak plasma in the first region of closed surfaces. The plasma boundary should join the lines intersecting at the $X$-point forming a figure-eight shaped separatrix. The separatrix region and, in particular, curves outside the separatrix are expected to become a chaotic field line region when a non-integrable perturbation is added. The circles, hyperbolae and lines must join smoothly at the connection points, insofar as their slopes are equal in each region [42].

This discretization procedure works well for points near the potential extrema, but it fails near the connection points $x_{A}$ and $x_{B}$. This is because the map equation used must be changed at the exact instant of transition from region to region. Consider, for example, the fixed point $(x, y)=$ $(c, 0)$, corresponding to the $X$-point of the single-null divertor. Placing an initial condition $\left(x_{0}, y_{0}\right)$ exactly on a line forming the separatrix we find that, after some iterations, the orbit crosses the connection point $x_{A}$ and would continue in a line. However, since it has entered the region $x<x_{A}$, the orbit should have followed a circle corresponding to the plasma boundary.

Hence, in order to have a discretization scheme which works near this connection point, we have to keep track of the time interval the orbit spends in each of the regions adjacent to $x=x_{A}$. Thus, we adjust the discretization period from $\Delta$ to the correct value for each region. Let $\left(x_{n}, y_{n}\right)$ and $\left(x_{n+1}, y_{n+1}\right)$ be the coordinates just before and just after traversing the connection point $x_{A}$, respectively. Starting from $\left(x_{n}, y_{n}\right)$, the time it takes for the trajectory to reach the connection 
point $x=x_{A}$, presumably less than $\Delta$, can be obtained as follows: the connection points coordinates $\left(x_{A}, y_{A}\right)$ are given, by equations (20) and (21), respectively, according to

$$
\begin{gathered}
x_{A}=\left(x_{n}-c\right) \cosh t_{A}+y_{n} \sinh t_{A}+c, \\
y_{A}=\left(x_{n}-c\right) \sinh t_{A}+y_{n} \cosh t_{A},
\end{gathered}
$$

where $t_{A}$ is the time elapsed between the map points $\left(x_{n}, y_{n}\right)$ and $\left(x_{A}, y_{A}\right)$, which follows by elimination from the above equations:

$$
t_{A}\left(x_{n}, y_{n}\right)=\cosh ^{-1}\left[\frac{\left(x_{A}-c\right)\left(x_{n}-c\right)-y_{A} y_{n}}{\left(x_{n}-c\right)^{2}-y_{n}^{2}}\right] .
$$

Moreover, we can eliminate $t_{A}$ and get the relation

$$
y_{A}=-\left[\left(x_{A}-c\right)^{2}-\left(x_{n}-c\right)^{2}+y_{n}^{2}\right]^{1 / 2} .
$$

Since the time it takes to go from $\left(x_{A}, y_{A}\right)$ to $\left(x_{n+1}, y_{n+1}\right)$ is $\Delta-t_{A}$, for the region $x<x_{A}$, equations (20) and (21) give

$$
\begin{gathered}
x_{n+1}=x_{A} \cos \left(\Delta-t_{A}\right)+y_{A} \sin \left(\Delta-t_{A}\right), \\
y_{n+1}=-x_{A} \sin \left(\Delta-t_{A}\right)+y_{A} \cos \left(\Delta-t_{A}\right) .
\end{gathered}
$$

The complete form of the integrable divertor map is presented in appendix A. In appendix B we show by direct calculation that the Jacobian of this map is equal to unity. Hence, with this procedure, the symplectic property of the map is not lost during the connections between different regions.

A similar procedure is followed for the other connection point $\left(x_{B}, y_{B}\right)$. In general, for each connection point, we have two time step adjustments, one for each sense of transition. For two connection points, as in the case of the single-null divertor map, we have seven map pieces: one for each region and four for the transitions between these regions. We note that, although the solutions for each region are simple, the matching requirements at each connection point make the problem highly nonlinear, since the coordinates and times for the connection points depend on the coordinates in a complicated way. In spite of this, in each region the map is both area preserving and integrable, the physical requirement required for a description of the field line structure. (see appendix A for the explicit form of the map for each of the seven regions.)

A Poincaré plot of the integrable map described above is shown in figure 3 for map parameters $a=0.235$ and $c=0.200$. Since the topology does not depend on the time step value, for now we fix $\Delta=1$ for all surfaces. We normalized the $x$ and $y$ values with respect to the minor radius of the Brazilian tokamak TCABR ( $b=0.22 \mathrm{~m}$, which is not to be confused with the potential adjustable parameter). The curves on the left side of $x_{A}$ and on the right side of $x_{B}$ are circles, and the curves between these points are hyperbolae.

\section{Application to a plasma column with an external conducting ring}

The method we are proposing for obtaining integrable maps uses a variable time step $\Delta$, related to the safety factor profile $q(r)$, which should be given a priori. In the case of a large aspect ratio tokamak in the absence of a poloidal divertor,

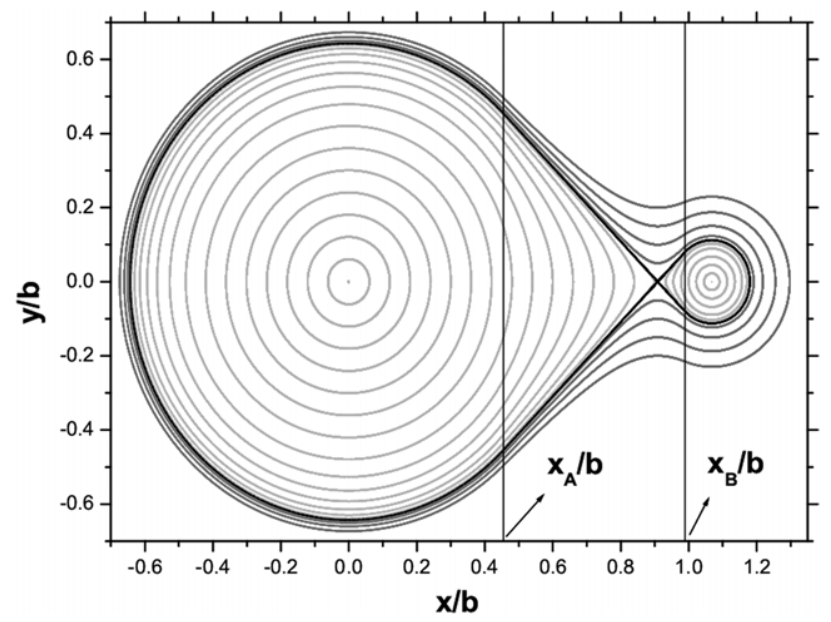

Figure 3. Invariant surfaces obtained from the integrable divertor map with the same time step $\Delta=1$ for all surfaces and parameters $c=0.200$ and $a=0.235$. The surfaces in light grey, black and dark grey are internal to the separatrix, on the separatrix and external to the separatrix, respectively.

where the cylindrical approximation $\left(r_{n}, \theta_{n}, z_{n}\right)$ is acceptable, the safety factor is given by

$$
q^{(\mathrm{p})}(r):=\frac{\mathrm{d} \phi}{\mathrm{d} \theta}=\frac{r}{R_{0}} \frac{B_{0}}{B_{\theta}^{(\mathrm{p})}(r)},
$$

where we used the magnetic field line equations (15) and assumed a cylindrical equilibrium tokamak field $\boldsymbol{B}^{(\mathrm{p})}=$ $\left(0, B_{\theta}^{(\mathrm{p})}(r), B_{0}\right)$. The superscript (p) refers to quantities related to a cylindrical plasma column.

Now, we assume a peaked plasma current profile with density $j=j_{z}(r) \hat{z}$, where

$$
j_{z}(r)=\frac{I_{\mathrm{P}}(\gamma+1)}{\pi a^{2}}\left(1-\frac{r^{2}}{a^{2}}\right)^{\gamma} \Theta(a-r),
$$

and where $I_{\mathrm{P}}$ is the total plasma current, $\Theta$ is the Heaviside function, $a$ is the cylindrical plasma radius (not to be confused with the potential parameter) and $\gamma$ is a constant ultimately determined by comparison with experiments. The equilibrium poloidal magnetic field can be obtained from Ampére's law, yielding

$$
B_{\theta}^{(\mathrm{p})}(r)=\frac{\mu_{0} I_{\mathrm{P}}}{2 \pi r}\left[1-\Theta(a-r)\left(1-\frac{r^{2}}{a^{2}}\right)^{\gamma+1}\right] .
$$

We have chosen parameters representative of the Brazilian tokamak TCABR [44]: major radius $R_{0}=0.61 \mathrm{~m}$, minor radius $b=0.22 \mathrm{~m}$, plasma radius $a=0.18 \mathrm{~m}$, plasma current $I_{\mathrm{P}}=65 \mathrm{kA}$, toroidal magnetic field at the magnetic axis $B_{0}=1.20 \mathrm{~T}$ and $\gamma=4.5$. The equilibrium poloidal magnetic field described above can be expressed in the rectangular coordinates $(x, y)$ as

$$
\begin{gathered}
B_{x}^{(\mathrm{p})}(x, y)=B_{\theta}^{(\mathrm{p})}(r) \frac{y}{\sqrt{x^{2}+y^{2}}}, \\
B_{y}^{(\mathrm{p})}(x, y)=-B_{\theta}^{(\mathrm{p})}(r) \frac{x}{\sqrt{x^{2}+y^{2}}},
\end{gathered}
$$

where $r=\sqrt{x^{2}+y^{2}}$ and $\tan \theta=y / x$. 

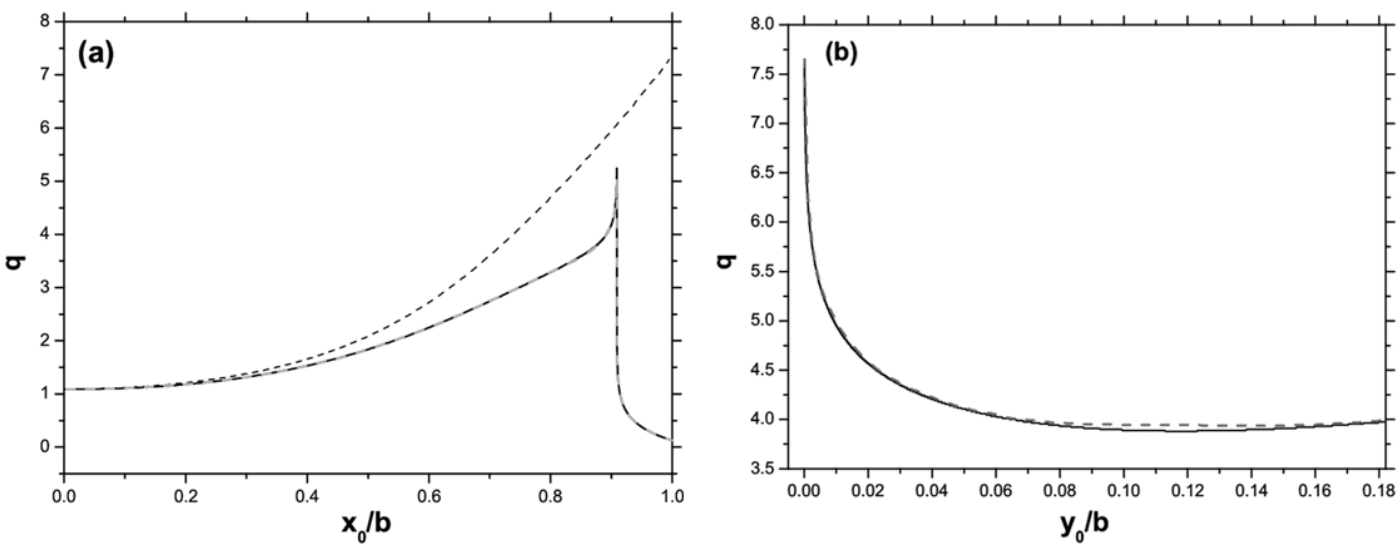

Figure 4. (a) Dependence of the safety factor with the horizontal initial conditions $(x, y)=\left(x_{0}, 0\right)$ for the plasma column (dashed black curve), the plasma plus a circular loop (solid black curve) and for the integrable divertor map using appropriate values for $\Delta(\psi)$ (dashed grey curve). (b) The same for the vertical initial conditions $(x, y)=\left(x_{\mathrm{S}}, y_{0}\right)$ ( $x_{\mathrm{S}}$ is the horizontal coordinate of the $X$-point) for the plasma plus a circular loop (solid black curve) and for the integrable divertor map using appropriate values for $\Delta(\psi)$ (dashed grey curve).

We design our single-null divertor with an $X$-point lying on the $x$-axis located at $(x, y)=\left(x_{\mathrm{S}}, 0\right)$. The closed magnetic surfaces in the region of the divertor will be produced by a concentric current ring at a position $(x, y)=\left(x^{\prime}, 0\right)$, carrying a current $I_{\mathrm{d}}$ in the $z$-direction. The field generated by this ring can be considered, in a cylindrical approximation, as the field of an infinite wire and is given, in rectangular coordinates, by

$$
\begin{aligned}
& B_{x}^{(\mathrm{d})}(x, y)=\frac{\mu_{0} y I_{\mathrm{d}}}{2 \pi\left[\left(x-x^{\prime}\right)^{2}+y^{2}\right]}, \\
& B_{y}^{(\mathrm{d})}(x, y)=\frac{-\mu_{0}\left(x-x^{\prime}\right) I_{\mathrm{d}}}{2 \pi\left[\left(x-x^{\prime}\right)^{2}+y^{2}\right]},
\end{aligned}
$$

where the superscript (d) refers to quantities related to the divertor current ring. We take the resultant field to be the linear superposition of the fields produced by the plasma cylindrical column and the current ring, which is acceptable insofar as we neglect the plasma response to the external magnetic field. The $X$-point occurs at the point where the resultant poloidal field is zero. So, making $B_{y}^{(\mathrm{p})}\left(x_{\mathrm{S}}, 0\right)=-B_{y}^{(\mathrm{d})}\left(x_{\mathrm{S}}, 0\right)$, we can obtain the expression

$$
\frac{I_{\mathrm{P}}}{I_{\mathrm{d}}}=\frac{x_{\mathrm{S}}}{\left(x^{\prime}-x_{\mathrm{S}}\right)},
$$

which relates the positions of the $X$-point and divertor ring with the plasma and divertor current. We imagine an $X$-point position between $x=a$ (the cylindrical plasma radius) and $x=b$ (the tokamak minor radius), i.e. $x_{\mathrm{S}}=0.2 \mathrm{~m}$. The external current ring position has a significant outward displacement with respect to the tokamak wall $x^{\prime}=0.235 \mathrm{~m}$. Substituting these values in (35), we find the value of an external current $I_{\mathrm{d}}=11.4 \mathrm{kA}$.

We numerically integrate the field line equations in order to study the magnetic field line dynamics in the presence of the external current ring:

$$
\begin{aligned}
& \frac{\mathrm{d} x}{\mathrm{~d} z}=\frac{B_{x}^{(\mathrm{p})}+B_{x}^{(\mathrm{d})}}{B_{0}}, \\
& \frac{\mathrm{d} y}{\mathrm{~d} z}=\frac{B_{y}^{(\mathrm{p})}+B_{y}^{(\mathrm{d})}}{B_{0}} .
\end{aligned}
$$

Solving the above equations we obtain the safety factor related to the initial condition used. The relation between the safety factor and initial conditions lying on the $x$-axis, for a cylindrical plasma in the presence of an external current ring, is depicted as the solid black curve in figure 4(a). The dashed black curve in figure 4(a) is obtained from equation (28) for the cylindrical plasma in the absence of the external current ring. The relation between the safety factor and the vertical initial conditions lying on $x=x_{\mathrm{S}}$ is depicted as the solid black curve in figure 4(b) for integration of (36) and (37).

Now, it remains to find the step size $\Delta$ for the map that matches the value of the function $\psi$, so as to apply the procedure explained in the previous section. However, the dependence of $\Delta$ on $q$ is not explicit, as it is for circular surfaces, and must be determined numerically, point by point, for several initial conditions, as presented in figures 4(a) and $(b)$. To compute $\psi$ for these initial conditions we test several values of $\Delta$ until we find one which presents the safety factor $q$, as close as possible to those shown in figures 4(a) and $(b)$. After that, we apply equations (9) and (19) to calculate $\psi\left(x_{0}, 0\right)$ or $\psi\left(x_{\mathrm{S}}, y_{0}\right)$, for horizontal or vertical initial conditions, respectively. The results of the step size $\Delta(\psi)$, for the three regions of the map, are displayed in figure 5 . The dependence of the safety factor on the initial conditions obtained for the integrable divertor map is depicted as the dashed grey curve in figures $4(a)$ and $(b)$, where we use the $\Delta$ values for the respective $\psi$ shown in figure 5 . We observe excellent agreement between the solid black curve (from numerical integration) and dashed grey curve (from the integrable map).

We choose surfaces of section with $z=2 \pi R_{0} n$, where $n$ is a positive integer, to plot the magnetic field lines for specified initial conditions. A comparison between magnetic surfaces obtained by numerical integration and those obtained from the integrable divertor map, with the same initial conditions for both cases, is shown in figure 6 . The vertical line indicates the position of the divertor plate. From the figure, it is seen that the map is not exact, but does a reasonable job of representing the surfaces. 


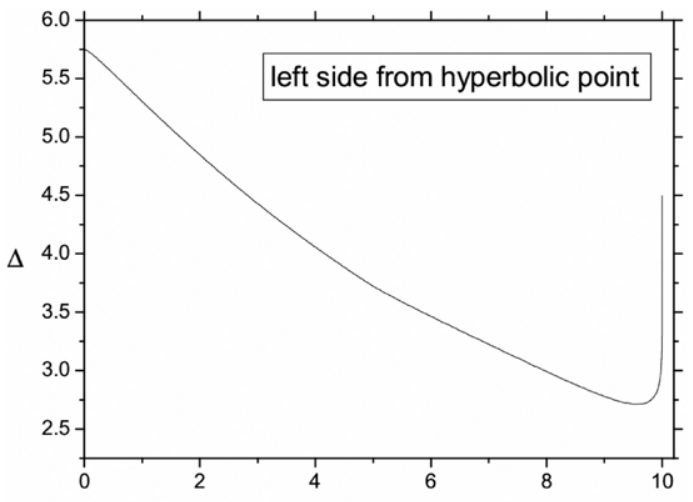

(a) $\Psi\left(10^{-3}\right)$
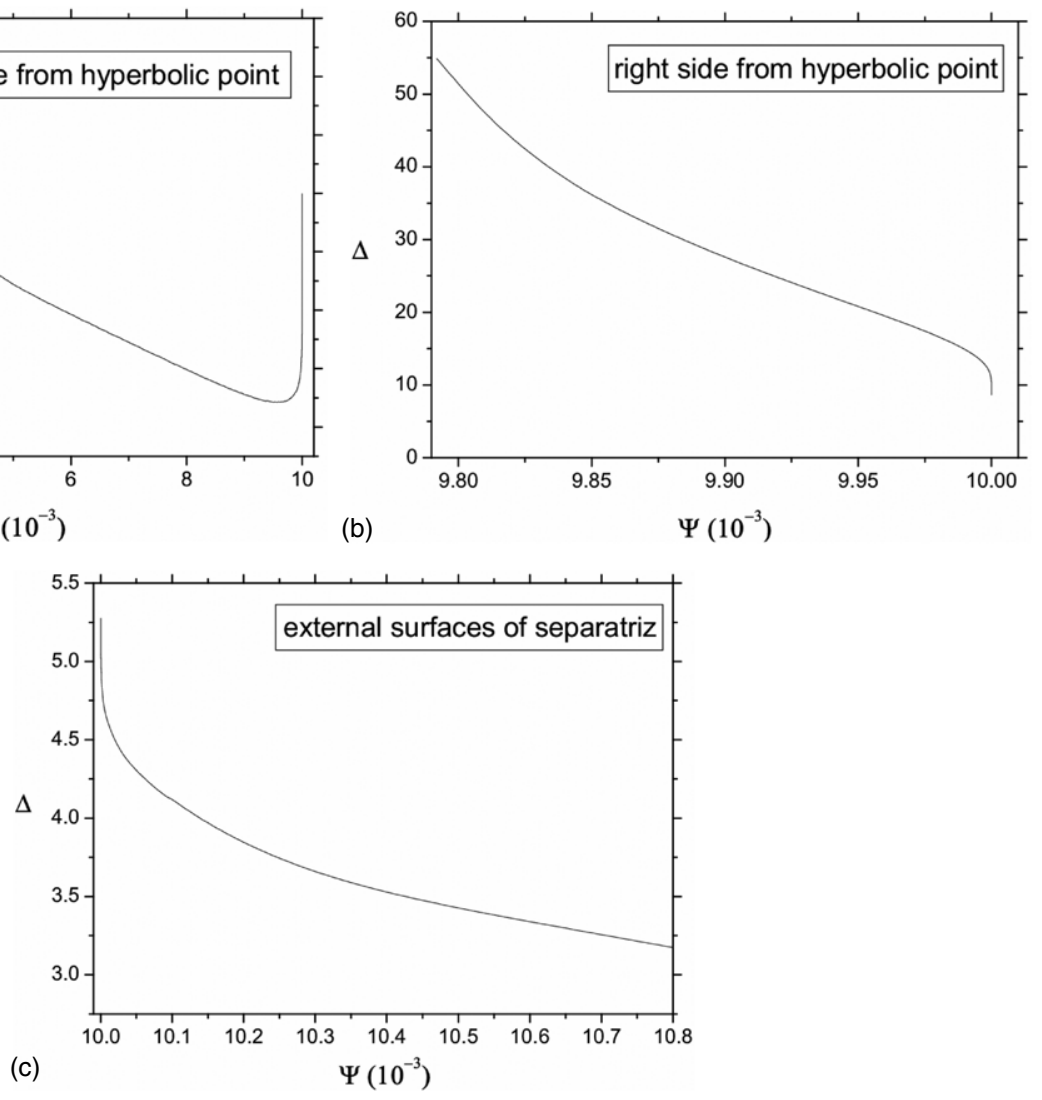

Figure 5. Relation between the step size $(\Delta)$ and the function $\psi$ used in the integrable divertor map in each of the three regions of phase space.

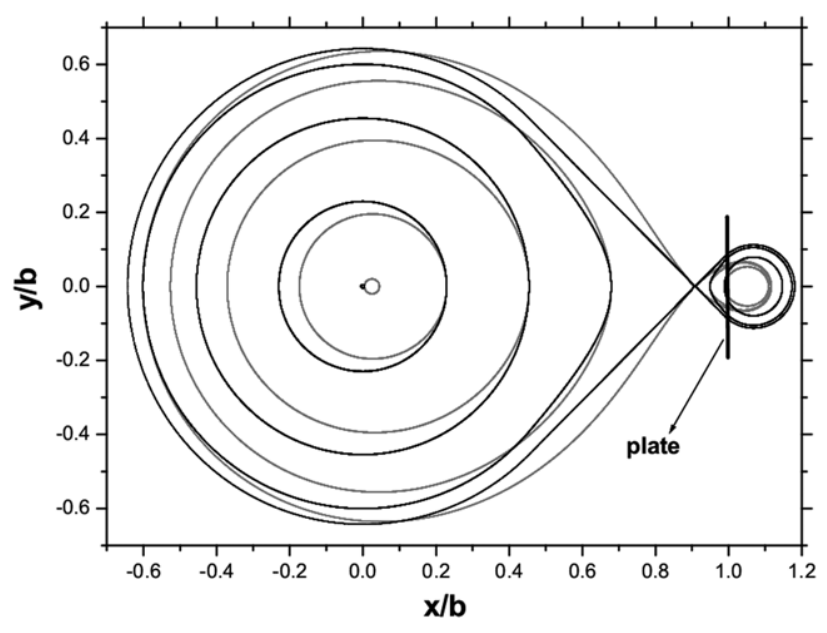

Figure 6. Magnetic surfaces for the numerical integration of field line equations of a circular plasma plus a current ring (grey) and for the integrable divertor map (black) with the same horizontal initial conditions.

\section{Divertor map with a non-integrable perturbation}

A main function of the divertor in a tokamak is to exhaust particles and energy from the plasma core in a controlled way. For that, it is necessary to have a peripheral region of chaotic magnetic field lines that comprise the plasma boundary and the $X$-point, and this chaotic region should intersect the divertor plate. Chaos in the tokamak peripheral region can be externally produced by an ergodic limiter, which is an arrangement of currents external to the plasma creating a perturbing magnetic field that is strong in the vicinity of the tokamak wall (figure 1). Hence, the creation of a region of chaotic field lines is most pronounced in the plasma edge and the scrape-off layer.

Many field line maps have been proposed to describe the formation of a chaotic region due to the presence of an ergodic limiter [13,45-47]. These models involve the composition of two maps, $\boldsymbol{M}=\boldsymbol{M}_{\mathrm{I}} \circ \boldsymbol{M}_{\mathrm{P}}$, where $\boldsymbol{M}_{\mathrm{I}}$ is an integrable map for describing the equilibrium dynamics and $\boldsymbol{M}_{\mathrm{P}}$ is a symmetrybreaking perturbative map due to the limiter currents. In this work, we take $M_{\mathrm{I}}$ to be the integrable divertor map obtained in the previous section.

The perturbation map, $\boldsymbol{M}_{\mathrm{P}}$, is obtained from the field generated by an ergodic limiter mounted over the tokamak chamber, designed as a ring-shaped coil of length $g$ and with $m$ pairs of straight sections in the toroidal direction, such that currents flow in opposite senses for two adjacent segments, as depicted in figure 1. Because the limiter is supposed to act only on the thin toroidal section it occupies (small $g$ ), limiters are similar to the kicked systems described in section 2. Thus, one expects a map to give reasonable results.

The perturbing map is written in the coordinates $(\rho, \alpha)$, which are related to the poloidal coordinates $(r, \theta)$ through $\rho=b-r$ and $\alpha=b \theta$, where $b$ is the minor radius of the tokamak chamber. According to the model proposed by Martin and Taylor [48], the field line entering the ergodic limiter at 

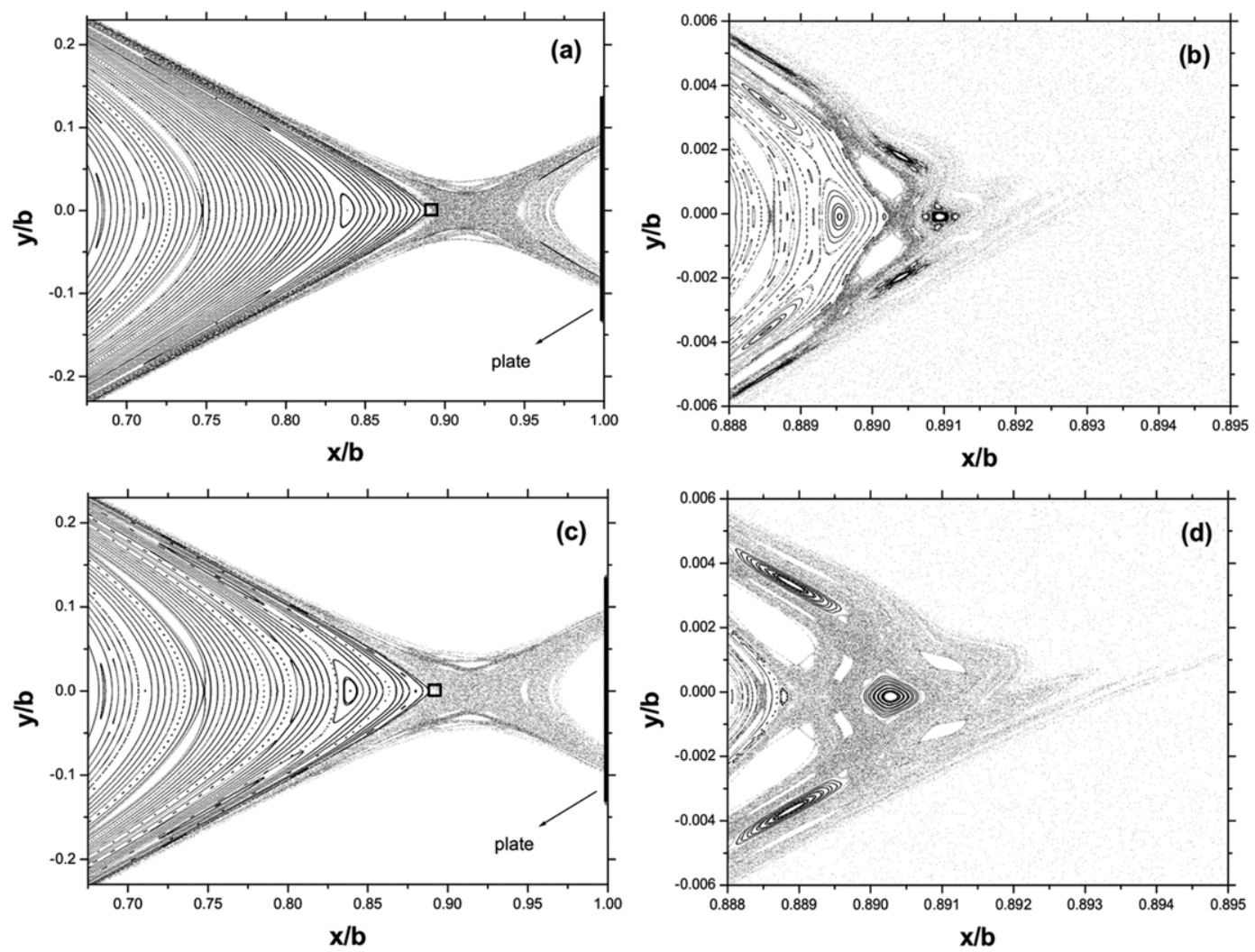

Figure 7. Poincaré maps for perturbation currents $(a) I_{\mathrm{h}}=0.20 \% I_{\mathrm{P}}$ and $(c) I_{\mathrm{h}}=0,31 \% I_{\mathrm{P}}$. In $(b)$ and $(d)$ are shown the amplification of the region indicated by a black square in $(a)$ and $(c)$ for the divertor plate, respectively. The initial conditions lie on the $x$-axis and coincide with the unperturbed separatrix. The black line represents a position imagined for the collector plate.

$\left(\rho_{1}, \alpha_{1}\right)$ emerges from it at $\left(\rho_{2}, \alpha_{2}\right)$ where

$\alpha_{2}=\alpha_{1}-C \mathrm{e}^{-m \rho_{1} / b} \cos \left(\frac{m \alpha_{1}}{b}\right)$,

$$
\begin{aligned}
\rho_{2}= & \rho_{1}+\frac{b}{m} \ln \left|\cos \left[\frac{m \alpha_{1}}{b}-\frac{m C}{b} \mathrm{e}^{-m \rho_{1} / b} \cos \left(\frac{m \alpha_{1}}{b}\right)\right]\right| \\
& -\frac{b}{m} \ln \left|\cos \left(\frac{m \alpha_{1}}{b}\right)\right|,
\end{aligned}
$$

and $C=\mu_{0} g m I_{\mathrm{h}} / \pi b B_{0}$, where $I_{\mathrm{h}}$ is the current flowing through the limiter segments, $b$ is the radial position of the ring and $B_{0}$ is the equilibrium toroidal field. In our simulations we use a limiter ring with length $g=0.08 \mathrm{~m}, m=5$ pairs of segment currents, the same toroidal magnetic field used in the previous section, $B_{0}=1.20 \mathrm{~T}$, and a limiter radial position coincident with the minor radius of tokamak chamber $b=0.22 \mathrm{~m}$. The number $m=5$ is chosen in order to cause a principal resonance in the external surfaces, with high safety factor.

The composition of the perturbing map $M_{\mathrm{P}}$ with the integrable divertor map $M_{\mathrm{I}}$ is performed as follows:

1. Calculate the value of $\psi$ for a field line position at iteration step $n$ from $\psi_{n}=\psi\left(x_{n}, y_{n}\right)=\frac{1}{2} y_{n}^{2}+V\left(x_{n}\right)$, and choose the $\Delta$ value that will be used in the next iteration of $M_{\mathrm{I}}$ through the relation $\Delta_{n}=\Delta\left(\psi_{n}\right)$, as given in figure 5;

2. Apply the integrable divertor map with step size $\Delta_{n}$, so as to find the position of the field line just before entering the limiter region, formally given by $\left(x^{*}, y^{*}\right)=M_{\mathrm{I}}\left(x_{n}, y_{n}\right)$;
3. Iterate the perturbing map $\left(x_{n+1}, y_{n+1}\right)=M_{\mathrm{P}}\left(x^{*}, y^{*}\right)$ (making the necessary changes of variables) for the field line to emerge from the limiter region. Repeat the steps above to get the next iteration of the composed map $M=M_{\mathrm{I}} \circ M_{\mathrm{P}}$.

The effect of $\boldsymbol{M}_{\mathrm{P}}$ is to cause a strong perturbation of the external surfaces, rendering the separatrix into a chaotic layer as can be seen in figures $7(a)$ and $(c)$, where we use the perturbation currents $I_{\mathrm{h}}=0.20 \% I_{\mathrm{P}}$ and $I_{\mathrm{h}}=$ $0.31 \% I_{\mathrm{P}}$, respectively. The initial conditions are taken on the $x$-axis, with maximum value coincident with the unperturbed separatrix $x_{S}$. Although there are no initial conditions on open surfaces, the Poincaré maps show that field lines are no longer confined to the closed surface region, which would happen for the unperturbed case. Instead, some lines escape, but always to the specific region of the divertor plate.

Because the safety factor goes to infinity as one approaches the separatrix, there is an accumulation of resonant modes in this region. This accumulation creates a large number of islands chains concentrated on a thin chaotic layer immersed in a chaotic sea, as we can see in figures $7(b)$ and $(d)$ (amplifications of the black squares are shown in figures 7(a) and $(c)$, respectively). We can see in figure $7(b)$ a concentration of points close to the last surviving island chain, which is due to a stickiness effect. The manifolds of this chain have an important influence on the escape of field lines to the plate. As the perturbation becomes larger, the last chain becomes totally destroyed and the next island chain emerges as the one responsible for stickiness, as shown in figure $7(d)$. The same 
effects were observed in recent work dealing with a tokamak with reversed magnetic shear and an ergodic limiter [14, 49].

\section{Traces of field line escape on the divertor plate}

The field line escape structure is closely related to the plasma particle deposition on the divertor plate, since we assume the particles follow the field lines as a guide. The map gives a field line position as it crosses the Poincaré section. However, to study field line escape we need to know where a field line reaches the divertor plate, which normally happens between iterations. To obtain the Poincaré map we discretized the continuous Hamiltonian system. However, we can use this fact to find the position of lines between iterations by an inverse procedure of turning the discrete map equations back into a continuous system for the one iteration before escape occurs.

The $x$-coordinate of the plate $\left(x_{\mathrm{p}}\right)$ is chosen as $x_{\mathrm{p}}=b$. Every time we have $x_{n}<x_{\mathrm{p}}$ and $x^{*}>x_{\mathrm{p}}$, we interpret this to mean the field line reaches the plate between these iterations. We use the continuous equations to find the final position $\left(x_{\mathrm{p}}, y_{\mathrm{F}}, t_{\mathrm{F}}\right)$ of the field line. Since $x_{\mathrm{p}}$ is greater than the connection point $x_{B}$, the final position of the field line is given, according to the continuous version of equations (20) and (21), by

$$
\begin{aligned}
& x_{\mathrm{p}}=\left(x_{n}-a\right) \cos t_{\mathrm{F}}+y_{n} \sin t_{\mathrm{F}}+a, \\
& y_{\mathrm{F}}=-\left(x_{n}-a\right) \sin t_{\mathrm{F}}+y_{n} \cos t_{\mathrm{F}},
\end{aligned}
$$

where $t_{\mathrm{F}}$ is the time elapsed between the map points $\left(x_{n}, y_{n}\right)$ and the final position $\left(x_{\mathrm{p}}, y_{\mathrm{F}}\right)$. From equations (40) and (41) we obtain the vertical position of the field line

$$
y_{\mathrm{F}}=\left[\left(x_{n}-a\right)^{2}-\left(x_{\mathrm{p}}-a\right)^{2}+y_{n}^{2}\right]^{1 / 2},
$$

and the time $t_{\mathrm{F}}$ as

$$
t_{\mathrm{F}}=\cos ^{-1}\left[\frac{\left(x_{p}-a\right)\left(x_{n}-a\right)+y_{\mathrm{F}} y_{n}}{\left(x_{n}-a\right)^{2}+y_{n}^{2}}\right] .
$$

The number of map iterations $N$ corresponds to the number of toroidal turns completed by the line before reaching the plate. The time $t_{\mathrm{F}}$ elapsed between the last iteration $n$ and the escape to the plate is related to the final toroidal angle $\Phi_{\mathrm{F}}$ for which the escape occurs. The value of $\Phi_{\mathrm{F}}$ is given by

$$
\Phi_{\mathrm{F}}=2 \pi \frac{t_{\mathrm{F}}}{\Delta_{n}} .
$$

The number of toroidal turns necessary for the field line to strike the divertor plate is also called the connection length $C_{\mathrm{L}}$, and is given by

$$
C_{\mathrm{L}}=N+\frac{t_{\mathrm{F}}}{\Delta_{n}},
$$

where $N$ is the number of map iterations.

\subsection{Connection lengths}

Specifically, we define the connection length to be the length of a field line (measured in number of toroidal turns) that begins at the lower part of the divertor plate (negative values of $y$ ) and ends at the upper part of the plate (positive values of $y$ ). We calculate the connection lengths of field lines for initial conditions on the divertor plate $\left(x_{0}=x_{\mathrm{p}}\right)$, in the vicinity of where the (unperturbed) separatrix intersects the divertor plate. The lines depart from the plate with negative $y$ values and strike the plate with positive $y$ values. The separatrix intersects the plate at two symmetric positions $y_{\mathrm{S}+}=0.019685$ and $y_{\mathrm{S}-}=-0.019685$. We take initial conditions close to $y_{\mathrm{S}-}$ and write $\Delta y_{0}=y_{0}-y_{\mathrm{S}-}$ as the displacement of initial condition with respect to the lower branch of which the unperturbed separatrix intersects the plate.

In figure $8(a)$ we see the connection lengths for the unperturbed case. The negative values of $\Delta y_{0}$ correspond to open surfaces external to the separatrix, while positive values of $\Delta y_{0}$ correspond to closed surfaces internal to the separatrix. The only high connection length occurs for the separatrix $\left(\Delta y_{0}=0\right)$, while all other field lines escape after few toroidal turns. In figure $8(b)$ we display the connection lengths for the perturbation parameter $I_{\mathrm{h}}=0.20 \% I_{\mathrm{P}}$ and in figure $8(c)$ for $I_{\mathrm{h}}=0.31 \% I_{\mathrm{P}}$. We observe a fractal-like structure in the dependence of the connection lengths on the initial conditions, as reported in [20].

This means that field lines with low connection lengths (less than, e.g. $\sim 10$ ) and very high connection lengths (more than $\sim 10^{3}$ for example) may have initial conditions that are very close. A large number of infinite connection lengths indicates a strong concentration of singular surfaces in a chaotic layer. Since, for the unperturbed case, the only singular surface is the separatrix, there is only one infinite connection length field line in figure $8(a)$. The perturbation causes the formation of a chaotic layer in the external region with a considerable number of island chains, each chain with its own singular surfaces. Consequently, we observe high connection lengths only for $\Delta y_{0}<0$, i.e. for initial conditions outside the unperturbed separatrix. The connection lengths obtained with $I_{\mathrm{h}}=0.31 \% I_{\mathrm{P}}$ (figure $8(b)$ ), are typically less than those with $I_{\mathrm{h}}=0.20 \% I_{\mathrm{P}}($ figure $8(c))$.

\subsection{Magnetic footprints}

Magnetic footprints are the set of strike points of field lines at the divertor plate, $\left(\Phi_{\mathrm{F}}, y_{\mathrm{F}}\right)$, which follows because the final horizontal coordinate is always $x_{\mathrm{p}}$; i.e. they correspond to marks on the divertor plate caused by lines that escape. The displacement $\Delta y_{\mathrm{F}}$ is measured from the point where the unperturbed separatrix intersects the plate, $\Delta y_{\mathrm{F}}=y_{\mathrm{F}}-y_{\mathrm{S}+}$. Positive values of $\Delta y_{\mathrm{F}}$ correspond to final vertical positions outside the unperturbed separatrix.

Figure $9(a)$ depicts footprints for the unperturbed case, without taking the final toroidal angle modulo $2 \pi$. In this case the value of $\Phi_{\mathrm{F}} / 2 \pi$ is equal to the connection length value. When we represent the same footprint with $\Phi_{\mathrm{F}}$ modulo $2 \pi$, as in figure $9(b)$, the plot typically overrides all the points between the dashed vertical lines in figure $9(a)$. The almost horizontal lines in figure $9(b)$ with $\Delta y_{\mathrm{F}} \sim 0$ are caused by lines close to the unperturbed separatrix on the inner and outer sides of it.

When the perturbation is taken into account, the structure in figure $9(b)$ becomes more complicated, as seen in figures $9(c)$ and $(d)$, for $I_{\mathrm{h}}=0.20 \% I_{\mathrm{P}}$ and $I_{\mathrm{h}}=0.31 \% I_{\mathrm{P}}$, respectively. The structure now occupies an area of the plate 

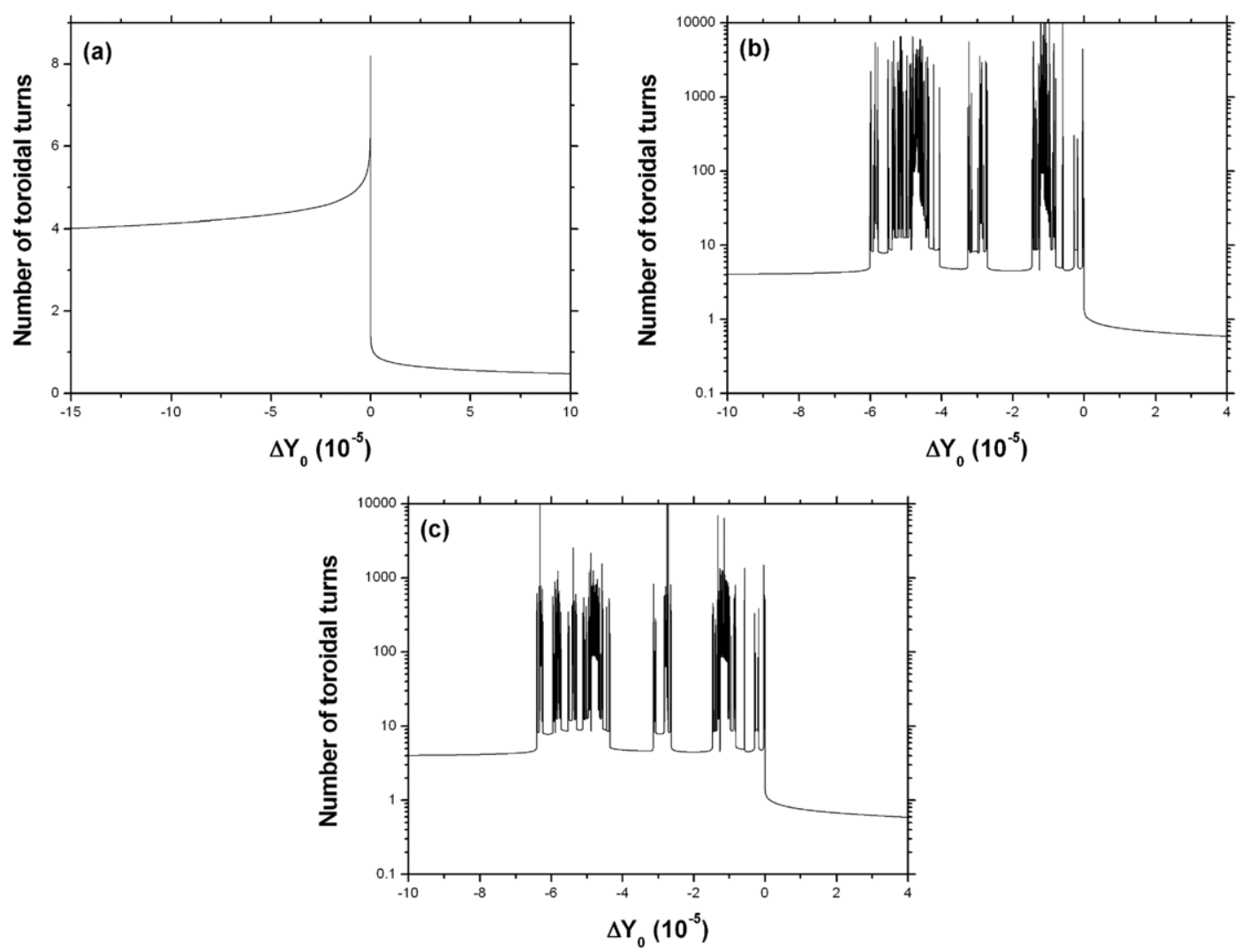

Figure 8. Connection lengths as a function of the displacement $\Delta y_{0}$ (with respect to $y_{\mathrm{s}^{-}}$) for (a) the unperturbed case, $(b) I_{\mathrm{h}}=0.20 \% I_{\mathrm{P}}$ and (c) $I_{\mathrm{h}}=0.31 \% I_{\mathrm{P}}$

with an apparent fractal structure. The continuity between $\Phi_{\mathrm{F}}=2 \pi$ and $\Phi_{\mathrm{F}}=0$ is no longer verified because of the impulsive kick-like perturbation. Moreover, upon increasing the perturbation parameter the footprint becomes wider, as seen by comparing figures $9(c)$ and $(d)$. This widening is a consequence of the chaotic layer enlargement shown in figure 7. This localized perturbation produced by an ergodic limiter gives rise to magnetic footprints rather different from those observed for equilibrium perturbed by continuous coils $[12,28,40,45]$.

\section{Conclusions}

In this paper we used a general method, presented in [42], which was developed to tailor-make integrable symplectic maps with specified fixed points in magnetostatic and Hamiltonian mechanics applications. The main points of this method were reviewed and applied to construct a symplectic poloidal divertor map. This integrable map describes the magnetic field line topology at the scrape-off layer of a large aspect ratio tokamak with a chosen safety factor profile and a poloidal divertor. We adjusted the time step parameter introduced in the divertor map according to the safety factor of the integrable flux surfaces. Results from our divertor map compare well with the Poincaré maps obtained through numerical integration of the field line equations.

We introduced external resonant non-axisymmetric magnetic perturbations, described by another symplectic map, in order to study flux surface breakup, and the corresponding connection lengths and the magnetic footprints on the divertor plate. The obtained divertor map is the composition of two symplectic maps: one that describes an MHD equilibrium with an $X$-point, whereas the other represents the resonant nonaxisymmetric perturbation that creates a chaotic layer around the outermost remaining flux surface.

\section{Acknowledgments}

This work was made possible through partial financial support from the following Brazilian research agencies: FAPESP (São Paulo), CAPES, CNPq and MCT-CNEN (Brazilian Fusion Network). P.J.M. was supported by the US Department of Energy Contract No DE-FG03-96ER-54346.

\section{Appendix A. Integrable divertor map}

The complete set of mapping equations for the integrable part of the divertor map is the following:

1. For $\left(x_{n}-c\right) \cosh \Delta+y_{n} \sinh \Delta+c<x_{A}<x_{n}$ :

$$
\begin{gathered}
x_{n+1}=x_{A} \cos \left(\Delta-t_{A}\right)+y_{A} \sin \left(\Delta-t_{A}\right), \\
y_{n+1}=-x_{A} \sin \left(\Delta-t_{A}\right)+y_{A} \cos \left(\Delta-t_{A}\right),
\end{gathered}
$$

where

$$
\begin{gathered}
t_{A}=\cosh ^{-1}\left[\frac{\left(x_{A}-c\right)\left(x_{n}-c\right)-y_{A} y_{n}}{\left(x_{n}-c\right)^{2}-y_{n}^{2}}\right], \\
y_{A}=-\left[\left(x_{A}-c\right)^{2}-\left(x_{n}-c\right)^{2}+y_{n}^{2}\right]^{1 / 2} .
\end{gathered}
$$



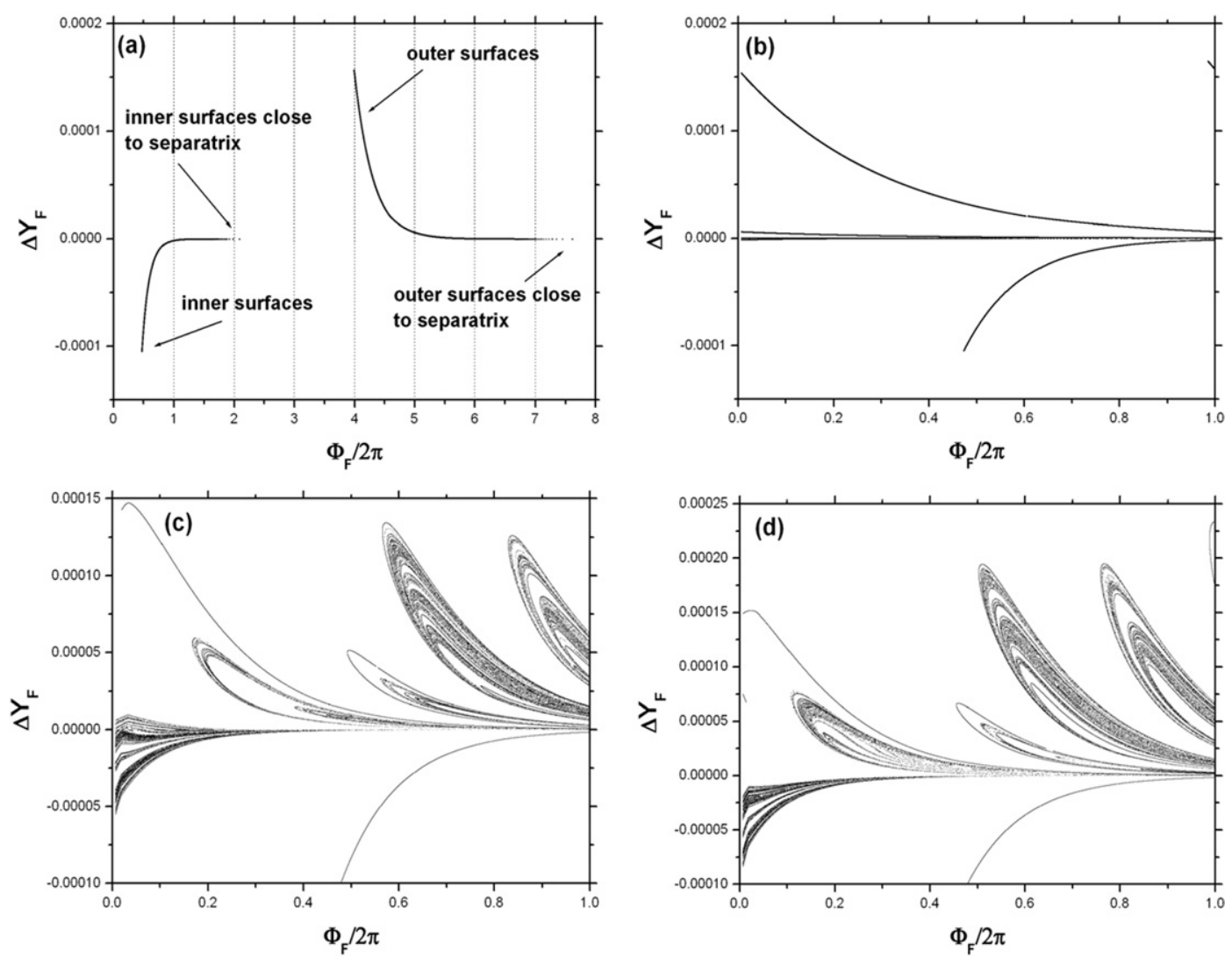

Figure 9. Magnetic footprints on the divertor plate for unperturbed case $(a)$ for the final angle of field lines $\Phi_{\mathrm{F}}$ non-modulo- $2 \pi$ and $(b)$ for $\Phi_{\mathrm{F}}$ modulo- $2 \pi$. For the perturbed case the magnetic footprints are shown with $\Phi_{\mathrm{F}}$ modulated for perturbation currents $(c) I_{\mathrm{h}}=0.20 \% I_{\mathrm{P}}$ and $(d) I_{\mathrm{h}}=0.31 \% I_{\mathrm{P}}$. The quantity $\left(\Delta y_{\mathrm{F}}\right)$ gives the displacement of the initial condition with respect to $y_{\mathrm{s}+}$.

2. For $x_{n}<x_{A}$ and $x_{n} \cos \Delta+y_{n} \sin \Delta<x_{A}$ :

$$
\begin{gathered}
x_{n+1}=x_{n} \cos \Delta+y_{n} \sin \Delta, \\
y_{n+1}=-x_{n} \sin \Delta+y_{n} \cos \Delta .
\end{gathered}
$$

3. For $x_{n}<x_{A}<x_{n} \cos \Delta+y_{n} \sin \Delta$

$$
x_{n+1}=\left(x_{A}-c\right) \cosh \left(\Delta-t_{A}^{*}\right)+y_{A}^{*} \sinh \left(\Delta-t_{A}^{*}\right)+c,
$$

$$
y_{n+1}=\left(x_{A}-c\right) \sinh \left(\Delta-t_{A}^{*}\right)+y_{A}^{*} \cosh \left(\Delta-t_{A}^{*}\right)
$$

where

$$
\begin{gathered}
t_{A}^{*}=\cos ^{-1}\left[\frac{x_{A} x_{n}+y_{A} y_{n}}{x_{n}^{2}+y_{n}^{2}}\right], \\
y_{A}^{*}=\left[x_{n}^{2}-x_{A}^{2}+y_{n}^{2}\right]^{1 / 2} .
\end{gathered}
$$

4. For $x_{A}<x_{n}<x_{B}$ and $x_{A}<\left(x_{n}-c\right) \cosh \Delta+y_{n} \sinh \Delta+$ $c<x_{B}$

$$
\begin{gathered}
x_{n+1}=\left(x_{n}-c\right) \cosh \Delta+y_{n} \sinh \Delta+c, \\
y_{n+1}=\left(x_{n}-c\right) \sinh \Delta+y_{n} \cosh \Delta .
\end{gathered}
$$

5. For $x_{n}<x_{B}<\left(x_{n}-c\right) \cosh \Delta+y_{n} \sinh \Delta+c$ :

$$
x_{n+1}=\left(x_{B}-a\right) \cos \left(\Delta-t_{B}\right)+y_{B} \sin \left(\Delta-t_{B}\right)+a \text {, }
$$

$$
y_{n+1}=-\left(x_{B}-a\right) \sin \left(\Delta-t_{B}\right)+y_{B} \cos \left(\Delta-t_{B}\right),
$$

where

$$
\begin{gathered}
t_{B}=\cosh ^{-1}\left[\frac{\left(x_{B}-c\right)\left(x_{n}-c\right)-y_{B} y_{n}}{\left(x_{n}-c\right)^{2}-y_{n}^{2}}\right], \\
y_{B}=\left[\left(x_{B}-c\right)^{2}-\left(x_{n}-c\right)^{2}+y_{n}^{2}\right]^{1 / 2} .
\end{gathered}
$$

6. For $x_{n}>x_{B}$ and $\left(x_{n}-a\right) \cos \Delta+y_{n} \sin \Delta+a>x_{B}$ :

$$
\begin{gathered}
x_{n+1}=\left(x_{n}-a\right) \cos \Delta+y_{n} \sin \Delta+a, \\
y_{n+1}=-\left(x_{n}-a\right) \sin \Delta+y_{n} \cos \Delta .
\end{gathered}
$$

7. For $\left(x_{n}-a\right) \cos \Delta+y_{n} \sin \Delta+a<x_{B}<x_{n}$ :

$x_{n+1}=\left(x_{B}-c\right) \cosh \left(\Delta-t_{B}^{*}\right)+y_{B}^{*} \sinh \left(\Delta-t_{B}^{*}\right)+c$,

$y_{n+1}=\left(x_{B}-c\right) \sinh \left(\Delta-t_{B}^{*}\right)+y_{B}^{*} \cosh \left(\Delta-t_{B}^{*}\right)$,

where

$$
\begin{aligned}
& t_{B}^{*}=\cos ^{-1}\left[\frac{\left(x_{B}-a\right)\left(x_{n}-a\right)+y_{B}^{*} y_{n}}{\left(x_{n}-a\right)^{2}+y_{n}^{2}}\right], \\
& y_{B}^{*}=-\left[\left(x_{n}-a\right)^{2}-\left(x_{B}-c\right)^{2}+y_{n}^{2}\right]^{1 / 2} .
\end{aligned}
$$




\section{Appendix B. Proof of the symplectic character of the map}

The Jacobian determinant of a 2D symplectic map must be equal to unity. In this appendix we show the symplectic character of the divertor map presented above. In order to shorten the exposition we will present these calculations only for two representative cases, namely those valid for region 1 and the connection between regions 2 and 1 .

\section{Appendix B.1. Map for region 1}

For points in region 1 which do not cross the frontier to region 2 we have, from equations (A5) and (A6), that the the Jacobian determinant, given by equation (3), is

$$
\operatorname{det}(J)=\frac{\partial x_{n+1}}{\partial x_{n}} \frac{\partial y_{n+1}}{\partial y_{n}}-\frac{\partial x_{n+1}}{\partial y_{n}} \frac{\partial y_{n+1}}{\partial x_{n}},
$$

and the partial derivatives are

$$
\begin{gathered}
\frac{\partial x_{n+1}}{\partial x_{n}}=\cos \Delta+\frac{\partial \Delta}{\partial x_{n}} K_{1}, \\
\frac{\partial x_{n+1}}{\partial y_{n}}=\sin \Delta+\frac{\partial \Delta}{\partial y_{n}} K_{1}, \\
\frac{\partial y_{n+1}}{\partial x_{n}}=-\sin \Delta+\frac{\partial \Delta}{\partial x_{n}} K_{2}, \\
\frac{\partial y_{n+1}}{\partial y_{n}}=\cos \Delta+\frac{\partial \Delta}{\partial y_{n}} K_{2},
\end{gathered}
$$

where

$$
\begin{aligned}
& K_{1}=-x_{n} \sin \Delta+y_{n} \cos \Delta, \\
& K_{2}=-x_{n} \cos \Delta-y_{n} \sin \Delta .
\end{aligned}
$$

On substituting (B2)-(B5) into (B1), the Jacobian determinant can be written as

$$
\begin{gathered}
\operatorname{det}(J)=1+\frac{\partial \Delta}{\partial x_{n}}\left(K_{1} \cos \Delta-K_{2} \sin \Delta\right) \\
+\frac{\partial \Delta}{\partial y_{n}}\left(K_{2} \cos \Delta+K_{1} \sin \Delta\right) \\
=1+y_{n} \frac{\partial \Delta}{\partial x_{n}}-x_{n} \frac{\partial \Delta}{\partial y_{n}} .
\end{gathered}
$$

The stepsize $\Delta$ can only be a function of $\psi_{n}$, and, since $\psi_{n}=y_{n}^{2} / 2+x_{n}^{2} / 2$, we have

$$
\begin{aligned}
& \frac{\partial \Delta}{\partial x_{n}}=\frac{\partial \Delta}{\partial \psi_{n}} \frac{\partial \psi_{n}}{\partial x_{n}}=x_{n} \frac{\partial \Delta}{\partial \psi_{n}}, \\
& \frac{\partial \Delta}{\partial y_{n}}=\frac{\partial \Delta}{\partial \psi_{n}} \frac{\partial \psi_{n}}{\partial y_{n}}=y_{n} \frac{\partial \Delta}{\partial \psi_{n}},
\end{aligned}
$$

such that the Jacobian determinant is

$$
\operatorname{det}(J)=1+x_{n} y_{n} \frac{\partial \Delta}{\partial \psi_{n}}-x_{n} y_{n} \frac{\partial \Delta}{\partial \psi_{n}}=1 .
$$

The Jacobian determinant for the other two maps which act in regions 2 and 3 without crossing the connection points, given by equations (A11)-(A12) and (A17)-(A18), respectively, can be found in a similar way to be equal to unity.
Appendix B.2. Map for the connection between regions 2 and 1

To map a point situated in region 2 at iteration $n$, to a position in region 1 at the iteration $n+1$ we use equations (A1) and (A2), where $y_{A}$ and $t_{A}$ are given, respectively, by equations (A3) and (A4). The partial derivatives appearing in the Jacobian determinant read as

$$
\begin{aligned}
& \frac{\partial x_{n+1}}{\partial x_{n}}=\frac{\partial x_{A}}{\partial x_{n}} \cos \left(\Delta-t_{A}\right)+\frac{\partial y_{A}}{\partial x_{n}} \sin \left(\Delta-t_{A}\right) \\
& +\left(\frac{\partial \Delta}{\partial x_{n}}-\frac{\partial t_{A}}{\partial x_{n}}\right) K_{1}^{\prime},
\end{aligned}
$$

$\frac{\partial x_{n+1}}{\partial y_{n}}=\frac{\partial x_{A}}{\partial y_{n}} \cos \left(\Delta-t_{A}\right)+\frac{\partial y_{A}}{\partial y_{n}} \sin \left(\Delta-t_{A}\right)$

$$
+\left(\frac{\partial \Delta}{\partial y_{n}}-\frac{\partial t_{A}}{\partial y_{n}}\right) K_{1}^{\prime},
$$

$\frac{\partial y_{n+1}}{\partial x_{n}}=-\frac{\partial x_{A}}{\partial x_{n}} \sin \left(\Delta-t_{A}\right)+\frac{\partial y_{A}}{\partial x_{n}} \cos \left(\Delta-t_{A}\right)$

$$
-\left(\frac{\partial \Delta}{\partial x_{n}}-\frac{\partial t_{A}}{\partial x_{n}}\right) K_{2}^{\prime},
$$

$$
\begin{aligned}
\frac{\partial y_{n+1}}{\partial y_{n}} & =-\frac{\partial x_{A}}{\partial y_{n}} \sin \left(\Delta-t_{A}\right)+\frac{\partial y_{A}}{\partial y_{n}} \cos \left(\Delta-t_{A}\right) \\
- & \left(\frac{\partial \Delta}{\partial y_{n}}-\frac{\partial t_{A}}{\partial y_{n}}\right) K_{2}^{\prime},
\end{aligned}
$$

with $K_{1}^{\prime}$ and $K_{2}^{\prime}$ given by

$$
\begin{gathered}
K_{1}^{\prime}=-x_{A} \sin \left(\Delta-t_{A}\right)+y_{A} \cos \left(\Delta-t_{A}\right), \\
K_{2}^{\prime}=x_{A} \cos \left(\Delta-t_{A}\right)+y_{A} \sin \left(\Delta-t_{A}\right) .
\end{gathered}
$$

On applying these relations in (3) and grouping the common terms, there results

$$
\begin{aligned}
\operatorname{det}(J) & =\frac{\partial x_{A}}{\partial x_{n}} \frac{\partial y_{A}}{\partial y_{n}}-\frac{\partial x_{A}}{\partial y_{n}} \frac{\partial y_{A}}{\partial x_{n}} \\
+ & \left(\frac{\partial \Delta}{\partial x_{n}}-\frac{\partial t_{A}}{\partial x_{n}}\right)\left(A \frac{\partial x_{A}}{\partial y_{n}}+B \frac{\partial y_{A}}{\partial y_{n}}\right) \\
- & \left(\frac{\partial \Delta}{\partial y_{n}}-\frac{\partial t_{A}}{\partial y_{n}}\right)\left(A \frac{\partial x_{A}}{\partial x_{n}}+B \frac{\partial y_{A}}{\partial x_{n}}\right),
\end{aligned}
$$

where

$$
\begin{gathered}
A=K_{2}^{\prime} \cos \left(\Delta-t_{A}\right)-K_{1}^{\prime} \sin \left(\Delta-t_{A}\right)=x_{A}, \\
B=K_{1}^{\prime} \cos \left(\Delta-t_{A}\right)+K_{2}^{\prime} \sin \left(\Delta-t_{A}\right)=y_{A},
\end{gathered}
$$

after having used equations (B17) and (B18).

Making use of definitions (22) and (23) and the relation $c=2 x_{A}$, the partial derivatives with respect to $x_{A}$ and $y_{A}$ can be written as

$$
\begin{aligned}
\frac{\partial x_{A}}{\partial x_{n}} & =\cosh t_{A}+y_{A} \frac{\partial t_{A}}{\partial x_{n}}, \\
\frac{\partial x_{A}}{\partial y_{n}} & =\sinh t_{A}+y_{A} \frac{\partial t_{A}}{\partial y_{n}}, \\
\frac{\partial y_{A}}{\partial x_{n}} & =\sinh t_{A}-x_{A} \frac{\partial t_{A}}{\partial x_{n}}, \\
\frac{\partial y_{A}}{\partial y_{n}} & =\cosh t_{A}-x_{A} \frac{\partial t_{A}}{\partial y_{n}},
\end{aligned}
$$


which, after substitution into the Jacobian determinant (B19), causes the vanishing of all the partial derivatives with relation to $t_{A}$, resulting in a simpler expression

$$
\begin{gathered}
\operatorname{det}(J)=1+\frac{\partial \Delta}{\partial x_{n}}\left(x_{A} \sinh t_{A}+y_{A} \cosh t_{A}\right) \\
-\frac{\partial \Delta}{\partial y_{n}}\left(x_{A} \cosh t_{A}+y_{A} \sinh t_{A}\right) .
\end{gathered}
$$

Now, given that $\Delta$ can only be a function of $\psi_{n}=$ $y_{n}^{2} / 2-\left(x_{n}-c\right)^{2} / 2+d$, we have

$$
\begin{gathered}
\frac{\partial \Delta}{\partial x_{n}}=\frac{\partial \Delta}{\partial \psi_{n}} \frac{\partial \psi_{n}}{\partial x_{n}}=-\left(x_{n}-c\right) \frac{\partial \Delta}{\partial \psi_{n}}, \\
\frac{\partial \Delta}{\partial y_{n}}=\frac{\partial \Delta}{\partial \psi_{n}} \frac{\partial \psi_{n}}{\partial y_{n}}=y_{n} \frac{\partial \Delta}{\partial \psi_{n}},
\end{gathered}
$$

such that, on applying these relations into equation (B26), we obtain

$$
\begin{gathered}
\operatorname{det}(J)=1-\frac{\partial \Delta}{\partial \psi_{n}}\left\{\left[y_{n} y_{A}+\left(x_{n}-c\right) x_{A}\right] \sinh t_{A}\right. \\
\left.+\left[y_{n} x_{A}+\left(x_{n}-c\right) y_{A}\right] \cosh t_{A}\right\} .
\end{gathered}
$$

The definitions of $x_{A}$ and $y_{A}$ given by (22) and (23), respectively, can be used once again in order to represent $\sinh t_{A}$ and $\cosh t_{A}$ in terms of $x_{A}, y_{A}, x_{n}$ and $y_{n}$, yielding

$$
\begin{aligned}
\sinh t_{A} & =\frac{y_{n}\left(x_{A}-c\right)-y_{A}\left(x_{n}-c\right)}{y_{n}^{2}-\left(x_{n}-c\right)^{2}}, \\
\cosh t_{A} & =\frac{\left(x_{n}-c\right)\left(x_{A}-c\right)-y_{A} y_{n}}{\left(x_{n}-c\right)^{2}-y_{n}^{2}},
\end{aligned}
$$

which, using $c=2 x_{A}$, make the term within braces in (B29) vanish identically, such that the Jacobian determinant results equal to unity. Similar calculations show that the maps for the other connections are also symplectic.

\section{References}

[1] Cordey J.G., Goldston R. J. and Parker R.R. 1992 Phys. Today 4522

[2] Post D.E. and the ITER TEAM 1991 ITER Documentation Series No 21 (Vienna: IAEA)

[3] Federici G. et al 2003 J. Nucl. Mater. 31311

[4] Portela J.S.E., Caldas I.L. and Viana R.L. 2008 Eur. Phys. J.: Special Top. 165195

[5] Boozer A. 2004 Rev. Mod. Phys. 761071

[6] Cary J. and Littlejohn R.G. 1983 Ann. Phys. (New York) 151

[7] Morrison P.J. 2000 Phys. Plasmas 72279

[8] Hinton F.L. and Hazeltine R.D. 1976 Phys. Fluids 172236

[9] Karger F. and Lackner K. 1975 Phys. Lett. A 61385

[10] Engelhardt W. and Feneberg W. 1978 J. Nucl. Mater. 76/77 518

[11] Abdullaev S.S. and Zaslavsky G.M. 1996 Phys. Plasmas 3516

[12] Abdullaev S.S., Jakubowski M., Lehnen M., Schmitz O. and Unterberg B. 2008 Phys. Plasmas 15042508

[13] da Silva E.C., Caldas I.L. and Viana R.L. 2001 Phys. Plasmas 82855
[14] Wingen A., Spatschek K.H. and Abdullaev S.S. 2005 Contrib. Plasma Phys. $\mathbf{4 5} 500$

[15] Wingen A., Jakubowski M., Spatschek K.H., Abdullaev S.S., Finken K.H. and Lehnen M. 2007 Phys. Plasmas 14042502

[16] da Silva E.C., Caldas I.L., Viana R.L. and Sanjuan M.A.F. 2002 Phys. Plasmas 94917

[17] Evans T.E., Moyer R.A. and Monat P. 2002 Phys. Plasmas 94957

[18] Borgogno D., Grasso D., Pegoraro F. and Schep T.J. 2008 Phys. Plasmas 15102308

[19] Boozer A.H. and Rechester A.B. 1978 Phys. Fluids 21682

[20] Reiman A. 1995 Phys. Plasmas 3906

[21] Pomphrey N. and Reiman A. 1992 Phys. Fluids B 4938

[22] Roeder R.K.W., Rapoport B.I. and Evans T.E. 2003 Phys. Plasmas 103796

[23] Spatschek K.H. 2008 Plasma Phys. Control. Fusion 50124027

[24] Caldas I.L. et al 2002 Braz. J. Phys. 32980

[25] Abdullaev S.S. 2006 Construction of Mappings for Hamiltonian Systems and their Applications (New York: Springer)

[26] Barocio S.R., Chávez-Alarcón E. and Gutierrez-Tapia C. 2006 Braz. J. Phys. 36550

[27] Punjabi A., Ali H., Evans T. and Boozer A. 2008 Phys. Plasmas 15082507

[28] Punjabi A. and Ali H. 2008 Phys. Plasmas 15122502

[29] Abdullaev S.S. and Finken K.H. 1998 Nucl. Fusion 38531

[30] Ali H., Punjabi A., Boozer A. and Evans T. 2004 Phys. Plasmas 111908

[31] Punjabi A., Verma A. and Boozer A. 1992 Phys. Rev. Lett. 693322

[32] Punjabi A., Verma A. and Boozer A. 1994 J. Plasma Phys. 5291

[33] Ali H., Punjabi A., Boozer A. and Evans T. 1994 Phys. Plasmas 111908

[34] Punjabi A., Verma A. and Boozer A. 1996 J. Plasma Phys. 56569

[35] Punjabi A., Ali H. and Boozer A. 1997 Phys. Plasmas 4337

[36] Punjabi A., Ali H. and Boozer A. 2003 Phys. Plasmas 103992

[37] Ali H. and Punjabi A. 2004 Phys. Plasmas 114527

[38] Abdullaev S.S. and Zaslavsky G.M. 1995 Phys. Plasmas 24533

[39] Abdullaev S.S. 2004 Phys. Rev. E 70046202

[40] Abdullaev S.S., Finken K.H., Jakubowski M. and Lehnen M. 2006 Nucl. Fusion 46 S113

[41] Abdullaev S.S. 2009 Phys. Plasmas 16030701

[42] Abbamonte P.M. and Morrison P.J. 1994 Constructing symplectic maps for application to magnetostatics and Hamiltonian mechanics Institute for Fusion Studies Report IFSR \# 638, The University of Texas at Austin, Austin, TX

[43] Lichtenberg A.J. and Lieberman M.A. 1992 Regular and Chaotic Motion 2nd edn (New York: Springer)

[44] Galvão R.M.O. et al 2001 Plasma Phys. Control. Fusion 431181

[45] Viana R.L. and Caldas I.L. 1992 Z. Naturf. A 47941

[46] Ullmann K. and Caldas I.L. 2000 Chaos Solitons Fractals 112129

[47] Evans T.E., DeGrassie J.S., Garner H.R., Leonard A.W., Ohyabu N. and Peranich L.S. 1989 J. Nucl. Mater. 162-164 636

[48] Martin T.J. and Taylor J.B. 1984 Plasma Phys. Control. Fusion 26321

[49] Kroetz T., Roberto M., da Silva E.C., Caldas I.L. and Viana R.L. 2008 Phys. Plasmas 15092310 